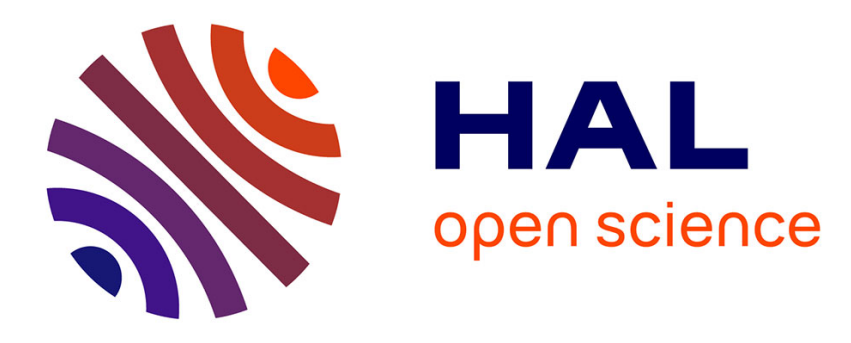

\title{
A Method of Piecewise-Smooth Numerical Branching
} Tomáš Ligurský, Yves Renard

\section{To cite this version:}

Tomáš Ligurský, Yves Renard. A Method of Piecewise-Smooth Numerical Branching. Journal of Applied Mathematics and Mechanics / Zeitschrift für Angewandte Mathematik und Mechanik, 2017, 97 (7), pp.815-827. 10.1002/zamm.201600219 . hal-01113564v4

\section{HAL Id: hal-01113564 \\ https://hal.science/hal-01113564v4}

Submitted on 14 Nov 2016

HAL is a multi-disciplinary open access archive for the deposit and dissemination of scientific research documents, whether they are published or not. The documents may come from teaching and research institutions in France or abroad, or from public or private research centers.
L'archive ouverte pluridisciplinaire HAL, est destinée au dépôt et à la diffusion de documents scientifiques de niveau recherche, publiés ou non, émanant des établissements d'enseignement et de recherche français ou étrangers, des laboratoires publics ou privés. 


\title{
A Method of Piecewise-Smooth Numerical Branching
}

\author{
Tomáš Ligurský ${ }^{1, *}$ and Yves Renard ${ }^{2}$ \\ ${ }^{1}$ Institute of Geonics of the Czech Academy of Sciences, Studentská 1768, 70800 Ostrava-Poruba, Czech Republic \\ ${ }^{2}$ Université de Lyon, CNRS, INSA-Lyon, ICJ UMR5208, F 69621, Villeurbanne, France
}

14 November 2016

Key words Numerical branching, piecewise smooth, steady-state problem, contact problem, Coulomb friction. MSC (2010) $65 \mathrm{P} 30$

\begin{abstract}
A method of numerical branching is proposed for piecewise-smooth steady-state problems when any analytical expressions are not known for the regions of smoothness of the function involved. The performance of the method is shown for model bifurcations in discretised contact problems with Coulomb friction. First, different parameter settings in its input are tested so that the optimum one can be proposed. Then, the method is used to investigate the behaviour of the bifurcations for different meshes. All solution branches seem to be discovered reliably if the parameters are set up properly.
\end{abstract}

\section{Introduction}

The steady-state bifurcation problem:

$$
\left.\begin{array}{l}
\text { Find } \boldsymbol{y} \in U \text { such that } \\
\boldsymbol{H}(\boldsymbol{y})=\mathbf{0}
\end{array}\right\}
$$

where $U \subset \mathbb{R}^{N+1}$ and $\boldsymbol{H}: U \rightarrow \mathbb{R}^{N}$, has been the subject of large number of studies in the last decades. A variety of numerical bifurcation methods has been constructed if $\boldsymbol{H}$ is smooth, say continuously differentiable (see, e.g., $[3,7,10]$ and references therein). On the other hand, there are many equilibrium problems in economics and diverse engineering fields whose models lead to a system of non-smooth equations $[2,6,18]$. Let us mention frictional and frictionless contact problems in solid mechanics, which are of our specific interest, for instance. However, methods of numerical branching of solutions of non-smooth problems depending on a parameter are still very little explored to our knowledge: Branching of static equilibrium curves of discretised frictionless contact problems were treated in $[4,19]$, where the tangential directions of curves emanating from points of non-smoothness were characterised as the solutions of a certain linear complementarity problem, and a method based on resolution of this problem was suggested for branch switching during numerical continuation (see also the theoretical survey [13]). Furthermore, the possible evolutions in quasi-static plane frictional contact problems were determined similarly in [16].

In our recent paper [14], we developed a restarted predictor-corrector method for numerical continuation of solution curves of Problem $(\mathscr{P})$ provided that $\boldsymbol{H}$ is piecewise $C^{1}\left(P C^{1}\right)$. The method consists in continuing smooth solution branches by a standard predictor-corrector method and joining the smooth branches continuously.

More precisely, our method computes a sequence of points $\left\{\boldsymbol{y}_{k}\right\}$ lying approximately on a selected solution branch together with a sequence of the corresponding tangent vectors $\left\{\boldsymbol{t}_{k}\right\}$ with a unit weighted norm $\left\|\boldsymbol{t}_{k}\right\|_{w}$. Starting from a couple $\left(\boldsymbol{y}_{k}, \boldsymbol{t}_{k}\right)$, one predictor-corrector step yields a new couple $\left(\boldsymbol{y}_{k+1}, \boldsymbol{t}_{k+1}\right)$ in the following way: In the predictor step, an initial approximation $(\tilde{\boldsymbol{y}}, \tilde{\boldsymbol{t}})$ of the new couple is generated in the direction of $\boldsymbol{t}_{k}$ as

$$
\tilde{\boldsymbol{y}}:=\boldsymbol{y}_{k}+h \boldsymbol{t}_{k}, \quad \tilde{\boldsymbol{t}}:=\boldsymbol{t}_{k},
$$

where $h$ is a step size. Then, the corrector steps, which are iterative steps of Newton's type, are run with the initial approximation $(\tilde{\boldsymbol{y}}, \tilde{\boldsymbol{t}})$. If they succeed in bringing the predicted point back to the currently approximated branch, the resulting couple is accepted, the step size $h$ is adapted for the next predictor-corrector step, and the current step is done. Otherwise, $h$ is reduced and the predictor and the corrector steps are repeated. If these predictor-corrector steps fail in computing a new couple, a special procedure is carried out for locating a new smooth branch (the so-called simple tangent

\footnotetext{
* Corresponding author, e-mail: tomas.ligursky@ugn.cas.cz, Phone: +420 596979326
} 


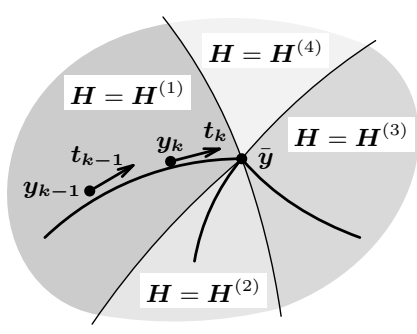

Fig. 1 An example of the considered situation.

switch). Afterwards, the predictor-corrector steps are restarted for continuation of the branch found. Let us emphasise that the whole method does not obey any analytical expressions of the regions of smoothness of $\boldsymbol{H}$, and it is implementable generically.

Similar continuation techniques constructed specially for static plane frictional contact problems can be found in [8,9]. Nevertheless, all these methods were proposed for finding solely one continuing branch when an end point of the most recently traced smooth branch is encountered. Besides, a bifurcation criterion was proposed merely in [15].

The present paper deals with purely non-smooth numerical branching of solutions of ( $\mathscr{P}$ ) when $\boldsymbol{H}$ is $P C^{1}$. We develop an approach that does not rely on any analytical expressions of the regions of smoothness of $\boldsymbol{H}$ in Section 2, and we test its performance on model examples of contact problems in Section 3.

Throughout the paper, we employ the following definition of a $P C^{1}$-function [18]:

Definition 1.1 A function $\boldsymbol{H}: U \rightarrow \mathbb{R}^{N}$ defined on an open set $U \subset \mathbb{R}^{M}$ is $P C^{1}$ if it is continuous and for every $\overline{\boldsymbol{y}} \in U$, there exist an open neighbourhood $O \subset U$ of $\overline{\boldsymbol{y}}$ and a finite family of $C^{1}$-functions $\boldsymbol{H}^{(i)}: O \rightarrow \mathbb{R}^{N}, i \in \mathcal{I}(\overline{\boldsymbol{y}})$, such that

$$
\forall \boldsymbol{y} \in O: \boldsymbol{H}(\boldsymbol{y}) \in\left\{\boldsymbol{H}^{(i)}(\boldsymbol{y}) ; i \in \mathcal{I}(\overline{\boldsymbol{y}})\right\}
$$

The functions $\boldsymbol{H}^{(i)}$ are termed selections of $\boldsymbol{H}$ at $\overline{\boldsymbol{y}}$.

\section{Numerical Branching}

Consider that an approximation of a smooth solution branch of $(\mathscr{P})$ has been obtained by the restarted predictor-corrector method from [14], and either the method has failed in locating a new smooth branch or it has located one but we want to find other ones because a bifurcation is expected by virtue of the criterion from [15], for example. Restricting ourselves to purely non-smooth branching (Assumptions (I)-(III) below), we shall describe a method designed for discovering potentially all continuing branches.

Let $\bar{y}$ be an end point of the recovered branch. We shall assume the following:

(I) The whole solution set of ( $\mathscr{P})$ in a vicinity of $\bar{y}$ is formed by one-sided smooth solution branches emanating from $\overline{\boldsymbol{y}}$ into mutually distinct regions of smoothness $\left\{\boldsymbol{y} \in O ; \boldsymbol{H}(\boldsymbol{y})=\boldsymbol{H}^{(i)}(\boldsymbol{y})\right\}$ for some $i \in \mathcal{I}(\overline{\boldsymbol{y}})$.

(II) The gradients $\boldsymbol{\nabla} \boldsymbol{H}^{(i)}(\overline{\boldsymbol{y}}), i \in \mathcal{I}(\overline{\boldsymbol{y}})$, have a full rank.

(III) $\operatorname{Ker} \boldsymbol{\nabla} \boldsymbol{H}^{(i)}(\overline{\boldsymbol{y}}) \cap \operatorname{Ker} \boldsymbol{\nabla} \boldsymbol{H}^{(j)}(\overline{\boldsymbol{y}})=\{\mathbf{0}\}, \quad \forall i, j \in \mathcal{I}(\overline{\boldsymbol{y}}), i \neq j$.

Note that these assumptions guarantee particularly that tangent vectors at $\bar{y}$ to any two different branches are linearly independent (see Fig. 1 for an illustration of branching under our consideration).

Since it seems to be hardly possible to encounter a point of non-smoothness in practical computations, we shall suppose in addition that $\boldsymbol{H}$ is smooth in any point considered in the procedures hereinafter. Then, a unit tangent vector $\boldsymbol{t}$ at such a point $\boldsymbol{y}$ lying in a vicinity of $\overline{\boldsymbol{y}}$ and belonging to a solution branch from the region $\left\{\boldsymbol{z} \in U ; \boldsymbol{H}(\boldsymbol{z})=\boldsymbol{H}^{(i)}(\boldsymbol{z})\right\}$ is uniquely determined up to a direction by the conditions:

$$
\boldsymbol{\nabla} \boldsymbol{H}^{(i)}(\boldsymbol{y}) \boldsymbol{t}=\mathbf{0}, \quad\|\boldsymbol{t}\|_{w}=1
$$

where $\|.\|_{w}$ denotes a suitable weighted norm [11, pp. 86 and 87].

Our branching method consists of two steps: an approximation of the end point $\overline{\boldsymbol{y}}$ and a subsequent location of new branches from a neighbourhood of the approximate end point.

\subsection{Approximation of the end point}

The end point can be approximated by a bisection-like procedure that is based on the predictor-corrector method used in the process of numerical continuation of smooth branches. The proposed procedure relies on the fact that the individual 


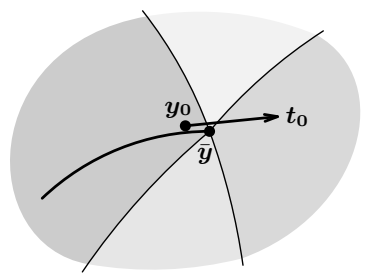

Fig. 2 Output of Algorithm 2.1.

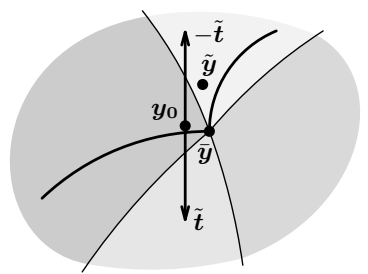

Fig. 3 Prediction direction for finding a new branch.

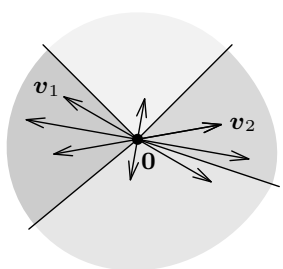

Fig. 4 Generation of directions into all regions.

branches emanating from the end point have numerically distinguishable tangents, which is justified by Assumptions (I)(III). The algorithm can be sketched as follows:

\section{Algorithm 2.1}

Input: $c_{\text {diff }} \in(0,1), h>h_{\min }>0, \boldsymbol{y}_{0}, \boldsymbol{t}_{0} \in \mathbb{R}^{N+1}$ such that

$$
\boldsymbol{H}\left(\boldsymbol{y}_{0}\right) \approx \mathbf{0}, \quad \boldsymbol{\nabla} \boldsymbol{H}\left(\boldsymbol{y}_{0}\right) \boldsymbol{t}_{0}=\mathbf{0},\left\|\boldsymbol{t}_{0}\right\|_{w}=1,
$$

and the corrector steps for the prediction $\tilde{\boldsymbol{y}}:=\boldsymbol{y}_{0}+h \boldsymbol{t}_{0}, \tilde{\boldsymbol{t}}:=\boldsymbol{t}_{0}$ do not converge or they converge to a couple $(\boldsymbol{y}, \boldsymbol{t})$ with $\boldsymbol{t}^{\top} \boldsymbol{t}_{0} /\left(\|\boldsymbol{t}\|\left\|\boldsymbol{t}_{0}\right\|\right)<c_{\text {diff }}$.

Step 1: Set $h:=h / 2$.

Step 2: Do the prediction $\tilde{\boldsymbol{y}}:=\boldsymbol{y}_{0}+h \boldsymbol{t}_{0}, \tilde{\boldsymbol{t}}:=\boldsymbol{t}_{0}$.

Step 3: Run the correction with $(\tilde{\boldsymbol{y}}, \tilde{\boldsymbol{t}})$.

Step 4: If the correction converges to a new couple $(\boldsymbol{y}, \boldsymbol{t})$ with $\boldsymbol{t}^{\top} \boldsymbol{t}_{0} /\left(\|\boldsymbol{t}\|\left\|\boldsymbol{t}_{0}\right\|\right) \geq c_{\mathrm{diff}}$, then set $\boldsymbol{y}_{0}:=\boldsymbol{y}, \boldsymbol{t}_{0}:=\boldsymbol{t}$.

Step 5: If $h<h_{\text {min }}$, break. Otherwise, go to Step 1.

Output: The point $\boldsymbol{y}_{0}$ with the corresponding unit tangent $\boldsymbol{t}_{0}$.

The couple $\left(\boldsymbol{y}_{0}, \boldsymbol{t}_{0}\right)$ in the input is supposed to be the last couple obtained by numerical continuation of the most recent smooth branch (the couple $\left(\boldsymbol{y}_{k}, \boldsymbol{t}_{k}\right)$ in Fig. 1), and $h$ equals typically the minimal step size from the predictor-corrector method employed during the continuation. We denote the Euclidean norm by $\|\cdot\|$ and $c_{\text {diff }}$ is the minimal value of the cosine of the angle of two tangent vectors considered to correspond to the same branch. Hence, if the conditions in Step 4 are satisfied, the newly found point $\boldsymbol{y}$ is supposed to be from the same branch as the one stored in $\boldsymbol{y}_{0}$ at that time, and it is accepted as a better approximation of $\overline{\boldsymbol{y}}$. The minimal step size $h_{\min }$ determines the precision required for the resulting approximation of $\overline{\boldsymbol{y}}$.

\subsection{Location of new branches}

Suppose that $\boldsymbol{y}_{0}$ calculated by Algorithm 2.1 is a good approximation of $\overline{\boldsymbol{y}}$, it belongs to some smooth branch, and $\boldsymbol{t}_{0}$ is the corresponding unit tangent pointing out from the region of smoothness with $\boldsymbol{y}_{0}$ (see Fig. 2). Inspired by the methods for branch switching at a smooth simple bifurcation point [7,10], we shall propose a heuristic technique composed of a sequence of predictor-corrector steps and yielding points on other smooth branches.

Our heuristic stems from the idea used in the simple tangent switch [14]: Let $\tilde{t}$ be a vector satisfying

$$
\boldsymbol{\nabla} \boldsymbol{H}(\tilde{\boldsymbol{y}}) \tilde{\boldsymbol{t}}=\mathbf{0}, \quad\|\tilde{\boldsymbol{t}}\|_{w}=1,
$$

where $\tilde{\boldsymbol{y}}$ is a point close to $\overline{\boldsymbol{y}}$ and lies in the region of smoothness containing a new smooth branch (Fig. 3). Then, either $\tilde{\boldsymbol{t}}$ or $-\tilde{t}$ should be a suitable prediction direction for a predictor-corrector step giving a point on this new branch.

Imagine for a while that you have a sequence of points $\tilde{\boldsymbol{y}}$ that pass through all regions of smoothness intersecting at $\overline{\boldsymbol{y}}$ at your disposal. Under Assumptions (I)-(III), you could discover all solution branches by computing the sequence of vectors $\tilde{\boldsymbol{t}}$ from (1) and trying the corresponding predictor-corrector steps with $\tilde{\boldsymbol{t}}$ and $-\tilde{\boldsymbol{t}}$ successively. Hence, we would like to guess such a sequence of points $\tilde{\boldsymbol{y}}$ from all the regions intersecting at $\overline{\boldsymbol{y}}$.

For this purpose, consider the most probable branching scenarios described in [15]: By linearisation and projection, the corresponding regions of smoothness can be represented by cones in a two-dimensional vector space with vertices at 
0. This representative space can be spanned by any two linearly independent vectors, say $\boldsymbol{v}_{1}$ and $\boldsymbol{v}_{2}$. Thus, a sequence of linear combinations of $\boldsymbol{v}_{1}$ and $\boldsymbol{v}_{2}$ whose directions are deployed densely enough in the space comprises the directions pointing into the representatives of all regions of smoothness as indicated in Fig. 4. From here, one can conclude that if $\boldsymbol{v}_{1}$ and $\boldsymbol{v}_{2}$ represent some vectors $\tilde{\boldsymbol{t}}_{1}$ and $\tilde{\boldsymbol{t}}_{2}$, then the corresponding sequence of linear combinations of $\tilde{\boldsymbol{t}}_{1}$ and $\tilde{\boldsymbol{t}}_{2}$ contains directions pointing into all regions and it can be used for calculating the sequence of points as desired.

In the general setting with $\left(\boldsymbol{y}_{0}, \boldsymbol{t}_{0}\right)$ from the output of Algorithm 2.1, let us choose a step length $h$ sufficiently large in comparison with the precision of the approximation of $\overline{\boldsymbol{y}}$ by $\boldsymbol{y}_{0}$, in particular so large that $\boldsymbol{y}_{0}+h \boldsymbol{t}_{0}$ leaves the region with $\boldsymbol{y}_{0}$. Next, let us take $\tilde{\boldsymbol{t}}_{1}:=-\boldsymbol{t}_{0}$ and calculate a vector $\tilde{\boldsymbol{t}}_{2}$ satisfying

$$
\nabla \boldsymbol{H}\left(\boldsymbol{y}_{0}+h \boldsymbol{t}_{0}\right) \tilde{\boldsymbol{t}}_{2}=\mathbf{0},\left\|\tilde{\boldsymbol{t}}_{2}\right\|_{w}=1 \text {. }
$$

According to the imposed assumptions, $\tilde{\boldsymbol{t}}_{1}$ and $\tilde{\boldsymbol{t}}_{2}$ are linearly independent. One can choose a set $\mathcal{B} \subset \mathbb{R}^{2}$ so that $\left\{\beta_{1} \tilde{\boldsymbol{t}}_{1}+\right.$ $\left.\beta_{2} \tilde{\boldsymbol{t}}_{2}\right\}_{\left(\beta_{1}, \beta_{2}\right) \in \mathcal{B}}$ is a sequence of densely deployed directions. With regard to the argumentation for the most probable branching scenarios, one can expect that the sequence $\left\{\boldsymbol{y}_{0}+h\left(\beta_{1} \tilde{\boldsymbol{t}}_{1}+\beta_{2} \tilde{\boldsymbol{t}}_{2}\right)\right\}\left(\beta_{1}, \beta_{2}\right) \in \mathcal{B}$ might be the desired one containing points from all regions intersecting at $\bar{y}$.

These considerations lead us to the following algorithm:

\section{Algorithm 2.2}

Input: $c_{\text {diff }} \in(0,1), n_{\text {dir }} \in \mathbb{N}, h, h_{\text {init }}>0, \boldsymbol{y}_{0}, \boldsymbol{t}_{0} \in \mathbb{R}^{N+1}$ such that

$$
\boldsymbol{H}\left(\boldsymbol{y}_{0}\right) \approx \mathbf{0}, \quad \boldsymbol{\nabla} \boldsymbol{H}\left(\boldsymbol{y}_{0}\right) \boldsymbol{t}_{0}=\mathbf{0},\left\|\boldsymbol{t}_{0}\right\|_{w}=1,
$$

and $\boldsymbol{y}_{0}+h \boldsymbol{t}_{0}$ leaves the region of smoothness with $\boldsymbol{y}_{0}$.

Step 1: Take $\tilde{\boldsymbol{t}}_{1}:=-\boldsymbol{t}_{0}$ and compute $\tilde{\boldsymbol{t}}_{2}$ such that

$$
\boldsymbol{\nabla} \boldsymbol{H}\left(\boldsymbol{y}_{0}+h \boldsymbol{t}_{0}\right) \tilde{\boldsymbol{t}}_{2}=\mathbf{0}, \quad\left\|\tilde{\boldsymbol{t}}_{2}\right\|_{w}=1 .
$$

Set $n_{\mathrm{br}}:=1, \boldsymbol{y}_{n_{\mathrm{br}}}:=\boldsymbol{y}_{0}, \boldsymbol{t}_{n_{\mathrm{br}}}:=\tilde{\boldsymbol{t}}_{1}$.

Step 2: Set $i:=0$.

\section{Step 3: Set}

$$
\alpha:=\frac{2 \pi i}{n_{\mathrm{dir}}}, \quad \boldsymbol{v}:=(\sin \alpha) \tilde{\boldsymbol{t}}_{1}+(\cos \alpha) \tilde{\boldsymbol{t}}_{2}, \quad \boldsymbol{v}:=\frac{\boldsymbol{v}}{\|\boldsymbol{v}\|_{w}},
$$

and compute $\tilde{t}$ such that

$$
\boldsymbol{\nabla} \boldsymbol{H}\left(\boldsymbol{y}_{0}+h \boldsymbol{v}\right) \tilde{\boldsymbol{t}}=\mathbf{0}, \quad\|\tilde{\boldsymbol{t}}\|_{w}=1
$$

Step 4: If $i=0$ or $\left|\tilde{\boldsymbol{t}}^{\top} \boldsymbol{t}_{0}\right| /\left(\|\tilde{\boldsymbol{t}}\|\left\|\boldsymbol{t}_{0}\right\|\right)<c_{\mathrm{diff}}$, set $\boldsymbol{t}_{0}:=\tilde{\boldsymbol{t}}$. Otherwise, go to Step 7 .

Step 5: Try one predictor-corrector step with the couple $\left(\boldsymbol{y}_{0}, \tilde{\boldsymbol{t}}\right)$ and the initial step size $h_{\text {init }}$. If it converges to a new couple $(\boldsymbol{y}, \boldsymbol{t})$, then

(i) if $\boldsymbol{t}^{\top}\left(\boldsymbol{y}-\boldsymbol{y}_{0}\right)<0$, take $\boldsymbol{t}:=-\boldsymbol{t}$;

(ii) if $\boldsymbol{t}^{\top} \boldsymbol{t}_{j} /\left(\|\boldsymbol{t}\|\left\|\boldsymbol{t}_{j}\right\|\right)<c_{\mathrm{diff}}, j=1, \ldots, n_{\mathrm{br}}$, then set $n_{\mathrm{br}}:=n_{\mathrm{br}}+1, \boldsymbol{y}_{n_{\mathrm{br}}}:=\boldsymbol{y}, \boldsymbol{t}_{n_{\mathrm{br}}}:=\boldsymbol{t}$.

Step 6: Repeat the procedure from Step 5 with $\tilde{t}:=-\tilde{t}$.

Step 7: Set $i:=i+1$. If $i<n_{\mathrm{dir}}$, go to Step 3. Otherwise, break.

Output: Points $\boldsymbol{y}_{1}, \ldots, \boldsymbol{y}_{n_{\mathrm{br}}}$ with the corresponding unit tangents $\boldsymbol{t}_{1}, \ldots, \boldsymbol{t}_{n_{\mathrm{br}}}$.

Here, $c_{\text {diff }}$ is the minimal absolute value of the cosine of the angle of two vectors from the kernels of gradients considered to correspond to the same selection similarly as in Algorithm 2.1. Further, $n_{\text {dir }}$ is the total number of linear combinations of $\tilde{\boldsymbol{t}}_{1}$ and $\tilde{\boldsymbol{t}}_{2}$ for seeking points from distinct regions. The step sizes $h$ and $h_{\text {init }}$ should be appropriately large in comparison with the expected precision of the approximation of $\overline{\boldsymbol{y}}$ by $\boldsymbol{y}_{0}$ so that the points determined with them fall into the regions in the respective directions. 
The conditions in Step 4 serve for determining whether $\tilde{\boldsymbol{t}}$ has been computed from a gradient from the same region as previously. If it is so, it is superfluous, hence the corresponding predictor-corrector steps are skipped. In Step 5(ii), one verifies whether $(\boldsymbol{y}, \boldsymbol{t})$ does not belong to any of the branches already found before. This ensures that the points in the output are from different smooth branches. The corresponding tangents are oriented in the directions of branching from $\overline{\boldsymbol{y}}$, which is guaranteed by Step 5(i). Afterwards, any of the branches located in this way can be traced by predictor-corrector steps.

Remark 2.3 To increase the probability of recovering all branches, one can restart Algorithm 2.2 several times from Step 2 with other choices of $\tilde{\boldsymbol{t}}_{1}$ and $\tilde{\boldsymbol{t}}_{2}$. We propose the following possibilities:

(i) Take $\tilde{\boldsymbol{t}}_{i}$ from the set $\left\{\boldsymbol{t}_{1}, \ldots, \boldsymbol{t}_{n_{\mathrm{br}}}\right\}$ at your current disposal.

(ii) Take $\tilde{\boldsymbol{t}}_{i}:=\tilde{\boldsymbol{t}}_{i}^{+}$with $\tilde{\boldsymbol{t}}_{i}^{+}$satisfying

$$
\boldsymbol{\nabla} \boldsymbol{H}\left(\boldsymbol{y}_{0}+h\left(\tilde{\boldsymbol{t}}_{i}^{-}+0.1 \frac{\tilde{\boldsymbol{t}}}{\|\tilde{\boldsymbol{t}}\|_{w}}\right)\right) \tilde{\boldsymbol{t}}_{i}^{+}=\mathbf{0}, \quad\left\|\tilde{\boldsymbol{t}}_{i}^{+}\right\|_{w}=1,
$$

where the value of $h$ is the same as in Algorithm 2.2, $\tilde{\boldsymbol{t}}_{i}^{-}$is equal to the vector $\tilde{\boldsymbol{t}}_{i}$ employed in the previous run of Algorithm 2.2 and $\tilde{t}$ is chosen randomly.

\section{Numerical Tests}

We have tested our technique on two examples of bifurcations in discretised elastostatic plane contact problems with Coulomb friction from [15]. We have chosen the following strategy for restarting Algorithm 2.2 based on Remark 2.3:

(1) Pass with $\tilde{\boldsymbol{t}}_{1}$ and $\tilde{\boldsymbol{t}}_{2}$ successively through the combinations from $\left\{\boldsymbol{t}_{1}, \ldots, \boldsymbol{t}_{n_{\mathrm{br}}}\right\}$ at your disposal.

(2) If all combinations which are available so far have already been employed, try to let $\tilde{\boldsymbol{t}}_{1}$ be the same and choose $\tilde{\boldsymbol{t}}_{2}$ according to Remark 2.3(ii).

In our tests, we denote the total number of selections of $\tilde{\boldsymbol{t}}_{1}$ and $\tilde{\boldsymbol{t}}_{2}$ by $n_{\mathrm{span}}$, that is, the total number of the restarts of Algorithm 2.2 equals $n_{\text {span }}-1$. Our calculations have been done with the finite-element library GetFEM++ [17].

\subsection{Elastostatic plane contact problems with Coulomb friction}

Let us consider static deformation of an elastic body whose reference configuration is the closure of a bounded domain $\Omega \subset \mathbb{R}^{2}$. Let the boundary $\partial \Omega$ be Lipschitz-continuous and split into three disjoint relatively open subsets $\Gamma_{D}, \Gamma_{N}$ and $\Gamma_{c}$. The body is fixed along $\Gamma_{D}$ whereas an applied surface force of density $\boldsymbol{h}$ is prescribed on $\Gamma_{N}$. A flat rigid foundation supports the body along $\Gamma_{c}$, and the contact is modelled by unilateral conditions and the Coulomb friction law. We suppose that there is no initial gap between the body and the foundation. We consider that the surface force at a point $\boldsymbol{x} \in \Gamma_{N}$ depends on a real parameter $\gamma$ and it may depend on $\boldsymbol{x}$ either, that is, $\boldsymbol{h}=\boldsymbol{h}(\gamma, \boldsymbol{x})$ in our model, $\gamma$ being a loading parameter. We seek equilibrium states of the body for the values of $\gamma$ from an interval $I$ of our interest.

Discretisation of this problem is done by applying a conforming Lagrange finite-element method to a mixed variational formulation of the problem with Lagrange multipliers enforcing the Dirichlet and the contact boundary conditions. The contact conditions are approximated nodally.

Let $\left\{\boldsymbol{\phi}_{i}\right\}_{1 \leq i \leq 2 n_{\Omega}}$ and $\left\{\boldsymbol{\xi}_{i}\right\}_{1 \leq i \leq 2 n_{D}}$ be bases of the shape functions of the finite elements for the displacement and the Lagrange multiplier corresponding to the Dirichlet condition, respectively. We introduce the following vectors and matrix:

$$
\begin{array}{ll}
\boldsymbol{A}(\boldsymbol{u})=\left(A_{i}(\boldsymbol{u})\right)_{1 \leq i \leq 2 n_{\Omega}}, & A_{i}(\boldsymbol{u}):=\int_{\Omega} \hat{\boldsymbol{\sigma}}\left(\boldsymbol{x}, \boldsymbol{I}+\sum_{j=1}^{2 n_{\Omega}} u_{j} \nabla \phi_{j}(\boldsymbol{x})\right): \nabla \boldsymbol{\phi}_{i}(\boldsymbol{x}) \mathrm{d} \boldsymbol{x}, \quad \boldsymbol{u}=\left(u_{j}\right)_{1 \leq j \leq 2 n_{\Omega}}, \\
\boldsymbol{L}(\gamma)=\left(L_{i}(\gamma)\right)_{1 \leq i \leq 2 n_{\Omega}}, & L_{i}(\gamma):=\int_{\Gamma_{N}} \boldsymbol{h}(\gamma, \boldsymbol{x}) \cdot \boldsymbol{\phi}_{i}(\boldsymbol{x}) \mathrm{d} s \\
\boldsymbol{B}_{D}=\left(B_{D, i j}\right)_{\substack{1 \leq i \leq 2 n_{D} \\
1 \leq j \leq 2 n_{\Omega}}}, \quad B_{D, i j}:=\int_{\Gamma_{D}} \boldsymbol{\xi}_{i}(\boldsymbol{x}) \cdot \boldsymbol{\phi}_{j}(\boldsymbol{x}) \mathrm{d} s .
\end{array}
$$

Here, $\hat{\sigma}$ stands for the first Piola-Kirchhoff stress tensor, which is replaced by the Cauchy stress tensor in the case of smalldeformation elasticity (the second example). Further, let $\left\{\boldsymbol{a}_{i}\right\}_{1 \leq i \leq n_{c}}$ be the set of the finite-element nodes corresponding to $\left\{\phi_{i}\right\}_{1 \leq i \leq 2 n_{\Omega}}$ and lying on $\bar{\Gamma}_{c} \backslash \bar{\Gamma}_{D}$, and $\nu$ and $\boldsymbol{\tau}$ denote the unit inward normal and the unit tangent to the foundation, respectively. One can define matrices $\boldsymbol{B}_{\nu}, \boldsymbol{B}_{\tau} \in \mathbb{R}^{n_{c} \times 2 n_{\Omega}}$ so that for any $\boldsymbol{u}^{h}=\sum_{j=1}^{2 n_{\Omega}} u_{j} \boldsymbol{\phi}_{j}$,

$$
\left(\boldsymbol{B}_{\nu} \boldsymbol{u}\right)_{i}=u_{\nu}^{h}\left(\boldsymbol{a}_{i}\right), \quad\left(\boldsymbol{B}_{\tau} \boldsymbol{u}\right)_{i}=u_{\tau}^{h}\left(\boldsymbol{a}_{i}\right), \quad i=1, \ldots, n_{c}
$$


where $u_{\nu}^{h}$ and $u_{\tau}^{h}$ are the components of $\boldsymbol{u}^{h}$ in the directions of $\boldsymbol{\nu}$ and $\boldsymbol{\tau}$ :

$$
u_{\nu}^{h}\left(\boldsymbol{a}_{i}\right)=\boldsymbol{u}^{h}\left(\boldsymbol{a}_{i}\right) \cdot \boldsymbol{\nu}, \quad u_{\tau}^{h}\left(\boldsymbol{a}_{i}\right)=\boldsymbol{u}^{h}\left(\boldsymbol{a}_{i}\right) \cdot \boldsymbol{\tau} .
$$

The discretised problem can be written in the following form of $(\mathscr{P})[1,12]$ :

$$
\left.\begin{array}{l}
\text { Find } \boldsymbol{y}:=\left(\gamma, \boldsymbol{u}, \boldsymbol{\lambda}_{D}, \boldsymbol{\lambda}_{\nu}, \boldsymbol{\lambda}_{\tau}\right) \in I \times \mathbb{R}^{2\left(n_{\Omega}+n_{D}+n_{c}\right)} \text { such that } \\
\boldsymbol{H}(\boldsymbol{y})=\mathbf{0},
\end{array}\right\}
$$

where $\boldsymbol{H}: I \times \mathbb{R}^{2\left(n_{\Omega}+n_{D}+n_{c}\right)} \rightarrow \mathbb{R}^{2\left(n_{\Omega}+n_{D}+n_{c}\right)}$ is defined by

$$
\boldsymbol{H}(\boldsymbol{y}):=\left(\begin{array}{c}
\boldsymbol{A}(\boldsymbol{u})-\boldsymbol{L}(\gamma)-\boldsymbol{B}_{D}^{\top} \boldsymbol{\lambda}_{D}-\boldsymbol{B}_{\nu}^{\top} \boldsymbol{\lambda}_{\nu}-\boldsymbol{B}_{\tau}^{\top} \boldsymbol{\lambda}_{\tau} \\
\boldsymbol{B}_{D} \boldsymbol{u} \\
-\frac{1}{r}\left(\lambda_{\nu, j}-\left(\left(\lambda_{\nu}-r \boldsymbol{B}_{\nu} \boldsymbol{u}\right)_{j}\right)_{-}\right), \quad j=1, \ldots, n_{c} \\
-\frac{1}{r}\left(\lambda_{\tau, j}-P_{\left[\mathscr{F}\left(\left(\lambda_{\nu}-r \boldsymbol{B}_{\nu} \boldsymbol{u}\right)_{j}\right)_{-},-\mathscr{F}\left(\left(\lambda_{\nu}-r \boldsymbol{B}_{\nu} \boldsymbol{u}\right)_{j}\right)_{-}\right]}\left(\left(\lambda_{\tau}-r \boldsymbol{B}_{\tau} \boldsymbol{u}\right)_{j}\right)\right), \quad j=1, \ldots, n_{c}
\end{array}\right)
$$

Here, $\boldsymbol{u} \in \mathbb{R}^{2 n_{\Omega}}$ is the vector of nodal displacements, $\boldsymbol{\lambda}_{D} \in \mathbb{R}^{2 n_{D}}$ is the Lagrange multiplier corresponding to the Dirichlet condition, and $\boldsymbol{\lambda}_{\nu}, \boldsymbol{\lambda}_{\tau} \in \mathbb{R}^{n_{c}}$ are the normal and tangential Lagrange multipliers on the contact zone, respectively. The notation (.) - means the negative part (or equivalently, the projection onto $(-\infty, 0])$, and $P_{[a, b]}($.$) stands for the projection$ onto an interval $[a, b], a \leq b$. The friction coefficient is represented by a non-negative constant $\mathscr{F}$ here and $r>0$ is an augmentation parameter. Since the projections onto an interval are $P C^{1}$-functions, $\boldsymbol{H}$ is also $P C^{1}$ provided that $\boldsymbol{A}$ and $\boldsymbol{L}$ are continuously differentiable.

Let us mention that if $h$ denotes the mesh size for the displacement, the parameter $r$ can be viewed as a coefficient of the proportionality between the Lagrange multipliers divided by $h$, which gives approximations of the contact stresses, and the displacements divided by $h$ on the contact zone (compare to the physical interpretation of the modulus of elasticity). Nevertheless, the (exact) solutions of the discrete problem do not depend on the value of $r$ because the formulations are mathematically equivalent for all $r>0$. Its choice affects only numerical solving of the problem.

\subsection{Problem with a rectangular body}

Consider deformation of a rectangular block that is $40 \mathrm{~mm}$ wide and $80 \mathrm{~mm}$ high, fixed along its upper side and loaded along its lateral sides (Fig. 5). A plane-strain approximation of the nonlinear Ciarlet-Geymonat constitutive law [5, Chapter 4] is used for the material of the block. Namely, the first Piola-Kirchhoff stress tensor $\hat{\sigma}$ is given by

$$
\begin{aligned}
\hat{\boldsymbol{\sigma}}(\boldsymbol{x}, \boldsymbol{F}) & =(\tilde{\boldsymbol{\sigma}}(\tilde{\boldsymbol{F}}))_{1 \leq i, j \leq 2}, \quad \tilde{\boldsymbol{F}}=\left(\begin{array}{cc}
\boldsymbol{F} & \mathbf{0} \\
\mathbf{0} & 1
\end{array}\right), \quad \boldsymbol{F} \in \mathbb{R}^{2 \times 2}, \\
\tilde{\boldsymbol{\sigma}}(\tilde{\boldsymbol{F}}) & =2 b\left(\operatorname{tr}\left(\tilde{\boldsymbol{F}}^{\top} \tilde{\boldsymbol{F}}\right)\right) \boldsymbol{I}+2\left(a-b \tilde{\boldsymbol{F}}^{\top}\right) \tilde{\boldsymbol{F}}+\left(2 c \operatorname{det}\left(\tilde{\boldsymbol{F}}^{\top} \tilde{\boldsymbol{F}}\right)-d\right) \tilde{\boldsymbol{F}}^{-\top}, \quad \tilde{\boldsymbol{F}} \in \mathbb{R}^{3 \times 3},
\end{aligned}
$$

where

$$
\lambda=4000 \mathrm{~N} / \mathrm{mm}^{2}, \quad \mu=120 \mathrm{~N} / \mathrm{mm}^{2}, \quad a=30 \mathrm{~N} / \mathrm{mm}^{2}
$$

and

$$
b=\frac{\mu}{2}-a, \quad c=\frac{\lambda}{4}-\frac{\mu}{2}+a, \quad d=\frac{\lambda}{2}+\mu .
$$

We have prescribed $\boldsymbol{h}(\gamma, \boldsymbol{x})=\gamma\left(-2,0.12\left(x_{1}-20\right)\right)$ (in N/mm ${ }^{2}$ ) on both lateral sides of the block, $r=10$ and $\mathscr{F}=1$. Discretisation is done by approximating the displacement and the Lagrange multiplier for the Dirichlet condition with continuous piecewise bilinear functions on a uniform mesh with 800 squares and 21 contact nodes.

There are six solution branches intersecting at $\gamma=0$, which are illustrated in Fig. 5. Branches 1,2 and 3 correspond to forcing the block to the right with no contact, contact-stick and contact-slip to the right of the lower right vertex of the block. Branches 4, 5 and 6, which are symmetric with respect to the axis $x_{1}=20$ of the block, correspond to forcing the block to the left with no contact, contact-stick and contact-slip to the left of the lower left vertex.

In the testing of our method, we have set $c_{\text {diff }}=0.99999, h=5 \mathrm{e}-7, h_{\min }=h / 2^{14} \doteq 3.1 \mathrm{e}-11$ and $h_{\text {init }}=5 \mathrm{e}-4$ in Algorithms 2.1 and 2.2. To start with, we have chosen various values of $n_{\mathrm{dir}}$, and we have looked into sufficient values of $n_{\text {span }}$ for finding all six branches. Our computations are summarised in Table 1, where the entry Input means the branch from which $\boldsymbol{y}_{0}$ is chosen in the input of Algorithm 2.1 (only the listings for Branches 1,2 and 3 are written down because 


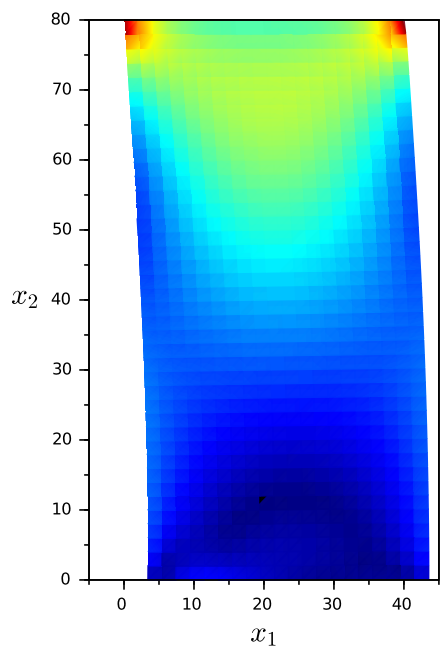

(a) Branch 1 .

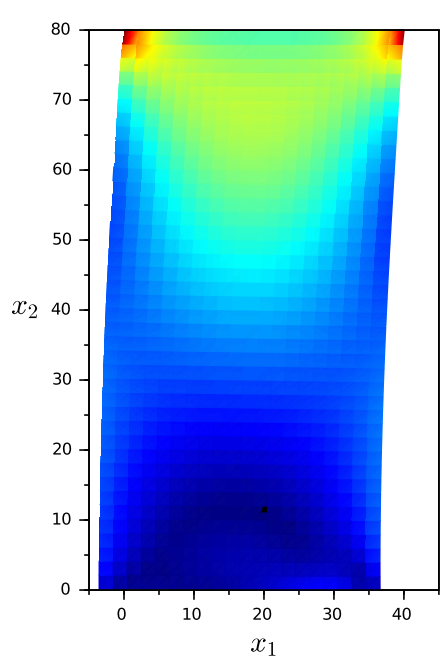

(d) Branch 4.

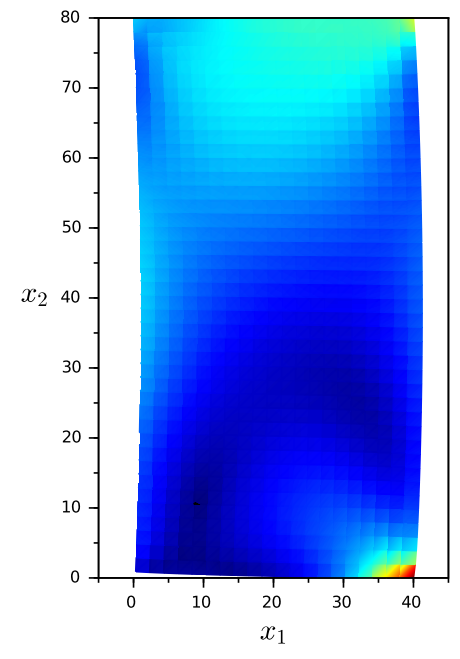

(b) Branch 2 .

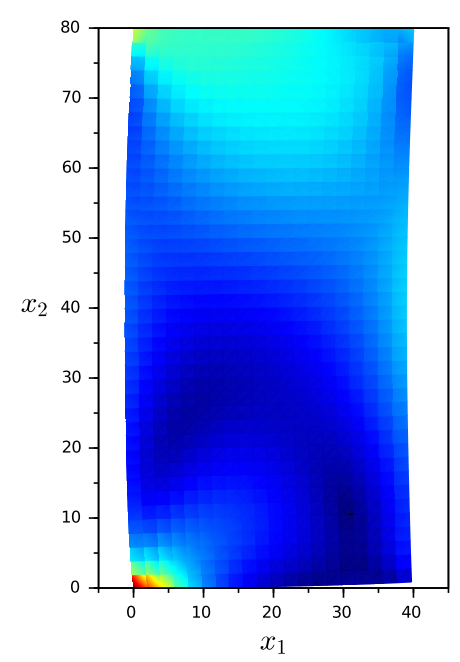

(e) Branch 5 .

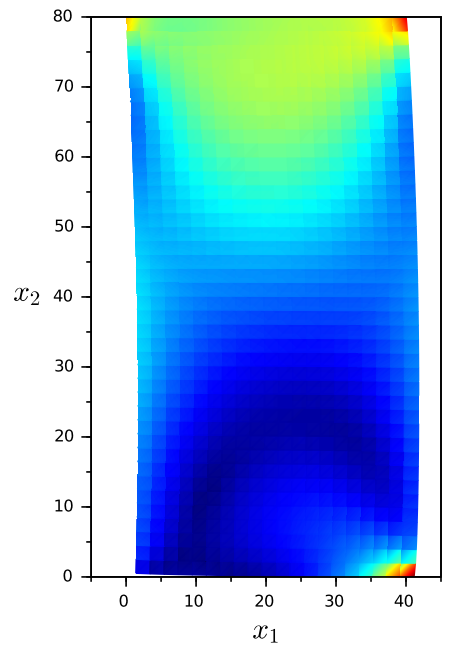

(c) Branch 3 .

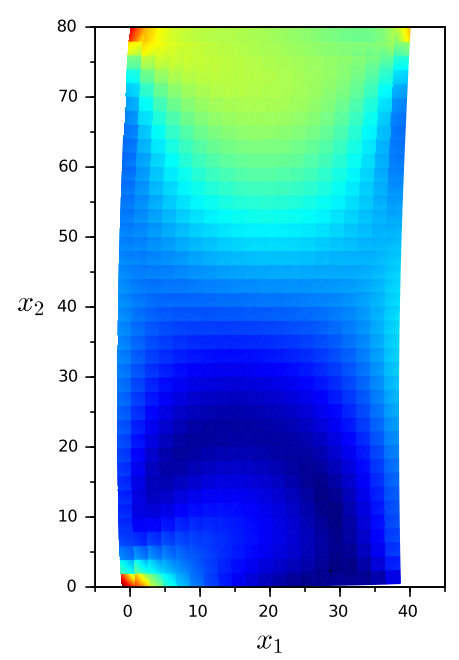

(f) Branch 6 .

Fig. 5 Deformed bodies corresponding to the solutions with $|\gamma|=1$ : (a), (b), (c) $\gamma=-1$; (d), (e), (f) $\gamma=1$.

the behaviour for Branches 4, 5 and 6 is the same due to the symmetry of the solutions). The cost of each computation is characterised by $n_{\mathrm{pc}}$, which stands for the total number of the predictor-corrector steps. These constitute the most expensive part of our technique. Note that random generations according to Remark 2.3(ii) are needed for some input branches and some values of $n_{\text {dir }}$. In such cases, we have run our branching method three times, and the behaviour of the performed computational process has varied from one run to another. We denote the total number of the restarts of Algorithm 2.2 following a random generation by $n_{\text {rand }}$ here and in what follows.

Afterwards, we have prescribed $n_{\mathrm{dir}}$ as well as $n_{\mathrm{span}}$, and we have compared the obtained values of $n_{\mathrm{pc}}$ to get an idea about the cheapest but quiet reliable strategy. The overview of the performance of our method is presented in Table 2 . All six branches have been recovered in all cases. According to the results, we can propose to set $n_{\text {dir }}$ to about 20 and $n_{\text {span }}$ to about 15 in this particular example. In addition, we can suggest to increase rather $n_{\text {dir }}$ than $n_{\text {span }}$ to increase the probability of finding all branches at the lowest price in general.

To explore the bifurcation phenomenon in the contact problem thoroughly, we have performed the method of numerical branching for discretisations on different uniform meshes, namely with 200, 800, 3200 and 12800 bilinear squares (M200, 
Table 1 Rectangular body, $n_{\text {dir }}$ given a priori.

\begin{tabular}{lrrrr}
\hline Input & $n_{\text {dir }}$ & $n_{\text {span }}$ & $n_{\text {pc }}$ & $n_{\text {rand }}$ \\
\hline \multirow{5}{*}{ Branch 1 } & 20 & 8 & 168 & 1 \\
& 20 & 10 & 214 & 3 \\
& 20 & 13 & 266 & 6 \\
\cline { 2 - 5 } & 40 & 7 & 292 & 2 \\
& 40 & 8 & 330 & 3 \\
\cline { 2 - 5 } & 50 & 14 & 478 & 7 \\
\cline { 2 - 5 } & 60 & 3 & 162 & 0 \\
\hline \multirow{5}{*}{ Branch 3 } & 5 & 232 & 0 \\
\cline { 2 - 5 } & 100 & 3 & 206 & 0 \\
\cline { 2 - 5 } & 20 & 5 & 128 & 0 \\
\cline { 2 - 5 } & 40 & 3 & 134 & 0 \\
\cline { 2 - 5 } & 60 & 1 & 72 & 0 \\
\hline
\end{tabular}

\begin{tabular}{lrrrr}
\hline Input & $n_{\text {dir }}$ & $n_{\text {span }}$ & $n_{\mathrm{pc}}$ & $n_{\text {rand }}$ \\
\hline & 20 & 4 & 136 & 1 \\
& 20 & 7 & 220 & 3 \\
& 20 & 8 & 240 & 4 \\
\cline { 2 - 5 } Branch 2 & 40 & 4 & 260 & 1 \\
& 40 & 5 & 294 & 2 \\
& 40 & 5 & 316 & 2 \\
\cline { 2 - 5 } & 50 & 4 & 274 & 1 \\
& 50 & 5 & 342 & 2 \\
& 50 & 7 & 490 & 4 \\
\cline { 2 - 5 } & 60 & 5 & 374 & 2 \\
& 60 & 8 & 620 & 5 \\
& 60 & 9 & 654 & 6 \\
\cline { 2 - 5 } & 100 & 4 & 372 & 1 \\
& 100 & 4 & 406 & 1 \\
& 100 & 7 & 662 & 4 \\
\hline
\end{tabular}

Table 2 Rectangular body, $n_{\text {dir }}$ and $n_{\text {span }}$ given a priori.

\begin{tabular}{lrrrr}
\hline Input & $n_{\text {dir }}$ & $n_{\text {span }}$ & $n_{\mathrm{pc}}$ & $n_{\text {rand }}$ \\
\hline \multirow{5}{*}{ Branch 1 } & 20 & 15 & 310 & 3 \\
& 20 & 15 & 316 & 5 \\
& 20 & 15 & 322 & 1 \\
\cline { 2 - 5 } & 20 & 16 & 326 & 3 \\
& 20 & 16 & 344 & 5 \\
& 50 & 16 & 346 & 1 \\
\cline { 2 - 5 } & 60 & 10 & 400 & 0 \\
\hline & 100 & 8 & 474 & 0 \\
\hline Branch 3 & 20 & 15 & 318 & 0 \\
& 20 & 15 & 318 & 0 \\
\cline { 2 - 5 } & 20 & 16 & 334 & 1 \\
& 20 & 16 & 334 & 1 \\
\cline { 2 - 5 } & 50 & 10 & 396 & 0 \\
\cline { 2 - 5 } & 60 & 10 & 414 & 0 \\
\hline & 100 & 8 & 456 & 0 \\
\hline
\end{tabular}

\begin{tabular}{lrrrr}
\hline Input & $n_{\text {dir }}$ & $n_{\text {span }}$ & $n_{\text {pc }}$ & $n_{\text {rand }}$ \\
\hline & 20 & 15 & 350 & 2 \\
& 20 & 15 & 360 & 4 \\
& 20 & 15 & 416 & 8 \\
\cline { 2 - 5 } Branch 2 & 20 & 16 & 374 & 2 \\
& 20 & 16 & 386 & 4 \\
& 20 & 16 & 434 & 8 \\
\cline { 2 - 5 } & 50 & 10 & 518 & 1 \\
& 50 & 10 & 532 & 1 \\
& 60 & 10 & 562 & 2 \\
\hline 60 & 10 & 618 & 2 \\
& 60 & 10 & 734 & 6 \\
\cline { 2 - 5 } & 100 & 8 & 642 & 1 \\
& 100 & 8 & 684 & 2 \\
& 100 & 8 & 720 & 3 \\
\hline
\end{tabular}

Table 3 The relative error of the displacements for $\gamma=-1$ for the rectangular body. The displacements $\boldsymbol{u}^{h_{1}}, \boldsymbol{u}^{h_{2}}, \boldsymbol{u}^{h_{3}}$ and $\boldsymbol{u}^{h_{4}}$ have been computed for M200, M800, M3200 and M12800, respectively.

\begin{tabular}{c|ccc}
\hline Branch & 1 & 2 & 3 \\
\hline$\left\|\boldsymbol{u}^{h_{1}}-\boldsymbol{u}^{h_{4}}\right\|_{1, \Omega} /\left\|\boldsymbol{u}^{h_{4}}\right\|_{1, \Omega}$ & $6.1 \mathrm{e}-2$ & $1.7 \mathrm{e}-1$ & $4.3 \mathrm{e}-2$ \\
$\left\|\boldsymbol{u}^{h_{2}}-\boldsymbol{u}^{h_{4}}\right\|_{1, \Omega} /\left\|\boldsymbol{u}^{h_{4}}\right\|_{1, \Omega}$ & $2.3 \mathrm{e}-2$ & $1.1 \mathrm{e}-1$ & $1.1 \mathrm{e}-2$ \\
$\left\|\boldsymbol{u}^{h_{3}}-\boldsymbol{u}^{h_{4}}\right\|_{1, \Omega} /\left\|\boldsymbol{u}^{h_{4}}\right\|_{1, \Omega}$ & $6.9 \mathrm{e}-3$ & $5.1 \mathrm{e}-2$ & $5.4 \mathrm{e}-3$ \\
\hline
\end{tabular}

M800, M3200 and M12800). Having taken $n_{\text {dir }}=30$ and $n_{\text {span }}=15$, we have found six branches distinctly separated for all meshes. The branches seem to be stable and to converge with the meshes, see the bifurcation diagrams in Fig. 6 obtained by the method of piecewise-smooth numerical continuation from [14], and the behaviour of the relative error for Branches 1, 2 and 3 in Table 3 (which is the same as the one for Branches 4, 5 and 6). 

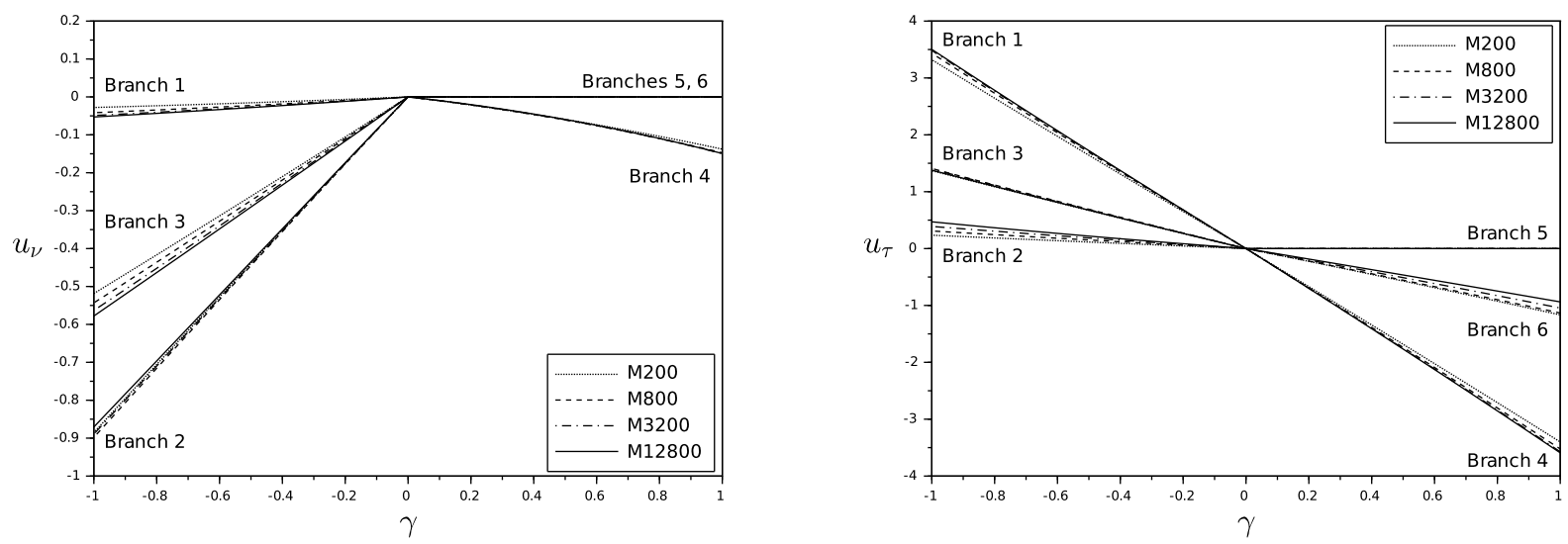

Fig. 6 The normal and tangential displacements $u_{\nu}$ and $u_{\tau}$ at the most left contact node of the rectangular body.

\subsection{Problem with a triangular body}

Next, let us consider deformation of an isosceles right-angled triangle with the legs $1 \mathrm{~m}$ long, lying on a leg, loaded along the other leg and fixed along the hypotenuse (Fig. 7). Let us restrict ourselves to the small-deformation framework with Hooke's law with the Lamé constants $\lambda=100 \mathrm{GN} / \mathrm{m}^{2}$ and $\mu=82 \mathrm{GN} / \mathrm{m}^{2}$, and let $r=10$ and $\mathscr{F}=1.7$. Both the displacement and the Lagrange multiplier for the Dirichlet condition are approximated with continuous piecewise linear functions over triangular meshes.

As described in [15], there are four solution branches intersecting at $\gamma=0$ for $\boldsymbol{h}=\boldsymbol{h}(\gamma)=\gamma(-26,-7.5) \mathrm{GN} / \mathrm{m}^{2}$ and the discretisation with a uniform mesh with 4096 triangles and 64 contact nodes, see Fig. 7 . They correspond to a partial contact and slip of the triangle to the right (Branch 2), and to no contact, contact-stick and contact-slip to the left of the lower left vertex of the triangle with pulling the whole triangle to the left (Branches 1, 3 and 4).

To explore the bifurcation in this contact problem, we have taken uniform meshes with 4096, 16384, 65536 and 262144 linear triangles (M4096, M16384, M65536 and M262144), and we have used our method with $c_{\text {diff }}=1-1 \mathrm{e}-10, h=$ $5 \mathrm{e}-8, h_{\text {min }}=h / 2^{14} \doteq 3.1 \mathrm{e}-12, h_{\text {init }}=5 \mathrm{e}-4, n_{\text {dir }}=30$ and $n_{\text {span }}=10$. The bifurcation behaviour has shown to be more complex here. Branches 1 and 4 approach one another for finer meshes, and they disappear both for the finest mesh for the force $\boldsymbol{h}(\gamma)=\gamma(-26,-7.5)$, as illustrated in Fig. 8 .

To investigate this behaviour more precisely, we have run an auxiliary numerical continuation of the same parametrised problem except the force $\boldsymbol{h}(\gamma)=(-20-10 \gamma,-7.5)$. As one can observe in Fig. 9, there are values of $\gamma$ for which the contact problem has multiple solutions for this parametrisation, but these values differ a bit for each mesh. Namely, the problem has three solutions for $\gamma$ approximately from $(0.56541,0.66665),(0.5883,0.70731),(0.59996,0.73289)$ and $(0.60585,0.7499)$ for M4096, M16384, M65536 and M262144, respectively. Moreover, two solutions approach one another when $\gamma$ approaches a bound of the interval corresponding to a fixed mesh.

Besides, note that $\boldsymbol{A}$ in (2) is linear in this case. If $\boldsymbol{h}$ is considered linear with respect to $\gamma$, then $\boldsymbol{L}$ is linear, as well, and $\boldsymbol{H}$ given by (2) is piecewise linear and especially positively homogeneous. This yields that the solutions obtained by the auxiliary continuation characterise the solution behaviour of the corresponding unparametrised contact problem with forces $\boldsymbol{h}=\left(h_{1}, h_{2}\right)$ for any $h_{2}<0$.

In particular, we have discovered three solutions of the unparametrised contact problem with forces $\boldsymbol{h}=\left(h_{1},-7.5\right)$ for $h_{1}$ approximately from $(25.6541,26.6665),(25.883,27.0731),(25.9996,27.3289)$ and $(26.0585,27.499)$ for the meshes M4096, M16384, M65536 and M262144, respectively. This and the positive homogeneity give us cones in the $h_{1}-h_{2}$ plane such that the unparametrised problem has three solutions for the forces from their interiors for each mesh. Furthermore, if a force approaches a half-line from the boundary of the cone for a given mesh, two of the three corresponding solutions approach one another.

Since the line $\boldsymbol{h}(\gamma)=\gamma(-26,-7.5)$ is closer and closer to a half-line from the boundaries of the cones for finer and finer meshes and out of the cone for the finest mesh, this explains the behaviour of the bifurcation in the present example. Nevertheless, regarding the branching in the whole $h_{1}-h_{2}$ plane, one can find it stable and convergent, again. This is illustrated by the bifurcation diagrams in Fig. 10 and Table 4 computed for a bit modified parametrised force $\boldsymbol{h}(\gamma)=\gamma(-26.4,-7.5)$, for which there are four branches distinctly separated for all meshes. 


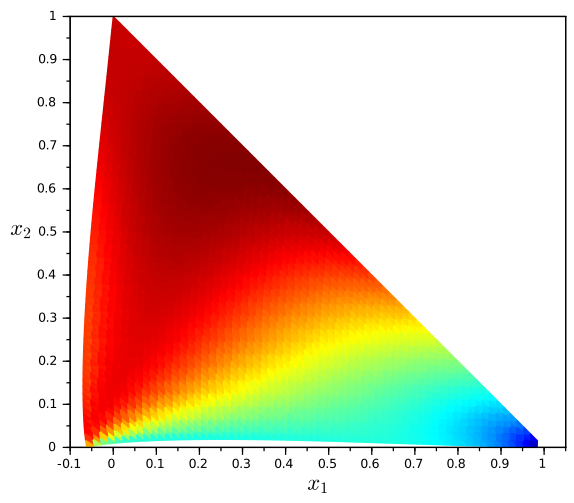

(a) Branch 1 .

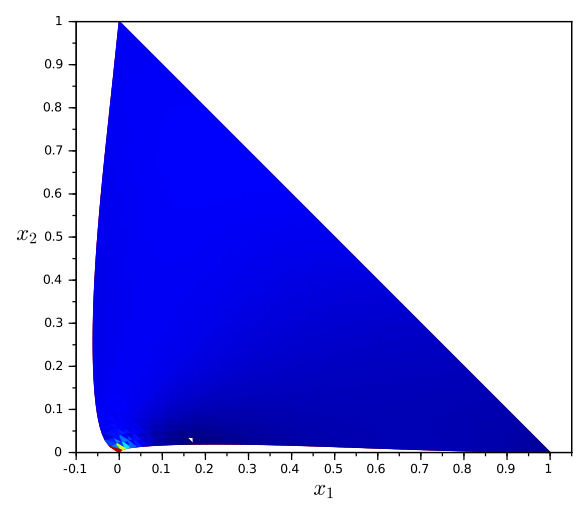

(c) Branch 3 .

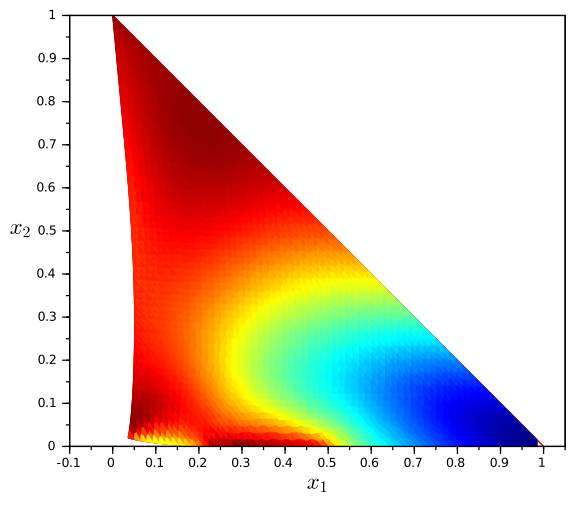

(b) Branch 2 .

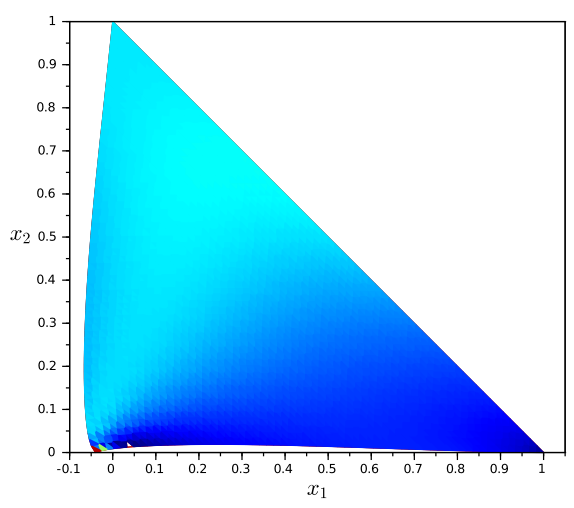

(d) Branch 4 .

Fig. 7 Deformed bodies corresponding to the solutions with $|\gamma|=1$ : (b) $\gamma=-1$; (a), (c), (d) $\gamma=1$.
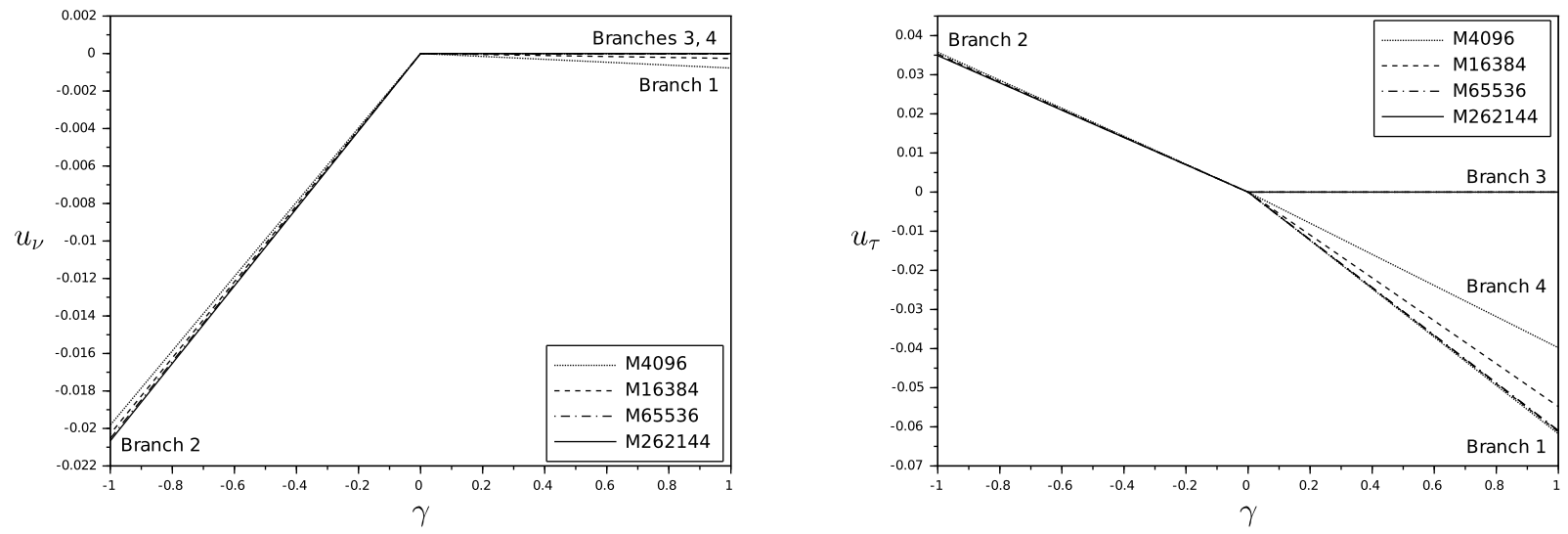

Fig. 8 The normal and tangential displacements $u_{\nu}$ and $u_{\tau}$ at the most left contact node of the triangular body for $\boldsymbol{h}(\gamma)=\gamma(-26,-7.5)$ (in $\mathrm{GN} / \mathrm{m}^{2}$ ). 

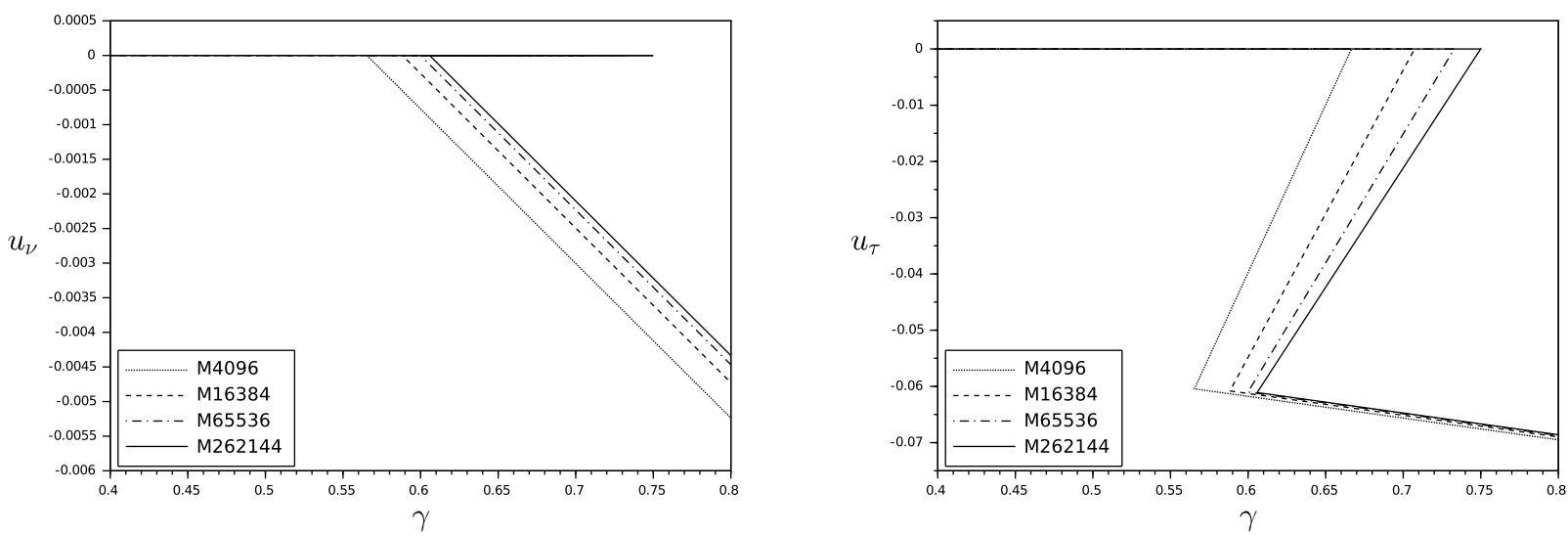

Fig. 9 The normal and tangential displacements $u_{\nu}$ and $u_{\tau}$ at the most left contact node of the triangular body for $\boldsymbol{h}(\gamma)=(-20-$ $10 \gamma,-7.5)\left(\right.$ in $\left.\mathrm{GN} / \mathrm{m}^{2}\right)$.
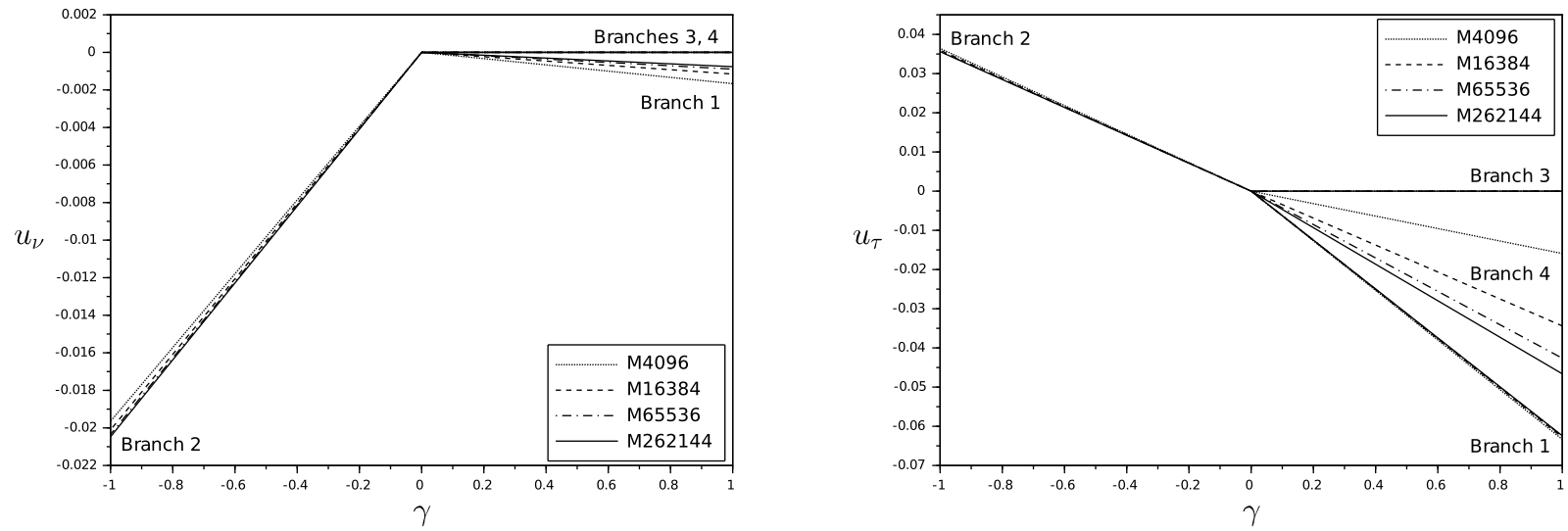

Fig. 10 The normal and tangential displacements $u_{\nu}$ and $u_{\tau}$ at the most left contact node of the triangular body for $\boldsymbol{h}(\gamma)=$ $\gamma(-26.4,-7.5)\left(\right.$ in $\left.\mathrm{GN} / \mathrm{m}^{2}\right)$.

Table 4 The relative error of the displacements for $\gamma=-1$ (Branch 2) and $\gamma=1$ (Branches 1, 3 and 4 ) for the triangular body. The displacements $\boldsymbol{u}^{h_{1}}, \boldsymbol{u}^{h_{2}}, \boldsymbol{u}^{h_{3}}, \boldsymbol{u}^{h_{4}}$ have been computed for M4096, M16384, M65536 and M262144, respectively.

\begin{tabular}{c|cccc}
\hline Branch & 1 & 2 & 3 & 4 \\
\hline$\left\|\boldsymbol{u}^{h_{1}}-\boldsymbol{u}^{h_{4}}\right\|_{1, \Omega} /\left\|\boldsymbol{u}^{h_{4}}\right\|_{1, \Omega}$ & $2.0 \mathrm{e}-2$ & $2.8 \mathrm{e}-2$ & $2.2 \mathrm{e}-1$ & $2.5 \mathrm{e}-1$ \\
$\left\|\boldsymbol{u}^{h_{2}}-\boldsymbol{u}^{h_{4}}\right\|_{1, \Omega} /\left\|\boldsymbol{u}^{h_{4}}\right\|_{1, \Omega}$ & $9.8 \mathrm{e}-3$ & $1.4 \mathrm{e}-2$ & $1.6 \mathrm{e}-1$ & $1.1 \mathrm{e}-1$ \\
$\left\|\boldsymbol{u}^{h_{3}}-\boldsymbol{u}^{h_{4}}\right\|_{1, \Omega} /\left\|\boldsymbol{u}^{h_{4}}\right\|_{1, \Omega}$ & $4.2 \mathrm{e}-3$ & $6.0 \mathrm{e}-3$ & $9.6 \mathrm{e}-2$ & $4.1 \mathrm{e}-2$ \\
\hline
\end{tabular}

\section{Conclusion}

We have developed a method of numerical branching for piecewise-smooth problems, and we have suggested how to choose its parameter setting to discover reliably all solution branches at the lowest price. We have used our method for investigating the behaviour of bifurcations in discretised contact problems with Coulomb friction for different meshes. As far as we know, this is the first attempt to devise a numerical method for piecewise-smooth branching that does not rely on any analytical expressions of the regions of smoothness of the function involved.

Acknowledgements The basis of this study was worked out at a postdoctoral stay of T. Ligurský at INSA-Lyon funded by Manufacture Française des Pneumatiques Michelin. During further developments, T. Ligurský was supported from the project "Support for building 
excellent research teams and inter-sectoral mobility at Palacký University in Olomouc II" (CZ.1.07/2.3.00/30.0041), and by The Ministry of Education, Youth and Sports of the Czech Republic from the National Programme of Sustainability (NPU II) project "IT4Innovations excellence in science - LQ1602".

\section{References}

[1] P. Alart and A. Curnier, A mixed formulation for frictional contact problems prone to Newton like solution methods, Comput. Methods Appl. Mech. Engrg. 92(3), 353-375 (1991).

[2] J. C. Alexander, T. Y. Li, and J. A. Yorke, Piecewise smooth homotopies, in: Homotopy methods and global convergence, edited by B. C. Eaves, F. J. Gould, H. O. Peitgen, and M. J. Todd (Plenum Press, New York, 1983), pp. 1-14.

[3] E. L. Allgower and K. Georg, Numerical path following, in: Techniques of Scientific Computing (Part 2), edited by P. G. Ciarlet and J. L. Lions, Handbook of numerical analysis Vol. V (Elsevier, 1997), pp. 3-207.

[4] G. Björkman, Path following and critical points for contact problems, Comput. Mech. 10(3-4), 231-246 (1992).

[5] P. G. Ciarlet, Mathematical elasticity, Vol. I: Three-dimensional elasticity, Studies in Mathematics and its Applications, Vol. 20 (North-Holland, Amsterdam, 1988).

[6] F. Facchinei and J. S. Pang, Finite-Dimensional Variational Inequalities and Complementarity Problems, Vol. I, Springer Series in Operations Research (Springer-Verlag, New York, 2003).

[7] K. Georg, Matrix-free numerical continuation and bifurcation, Numer. Funct. Anal. Optim. 22(3-4), 303-320 (2001).

[8] J. Haslinger, V. Janovský, and R. Kučera, Path-following the static contact problem with Coulomb friction, in: Proceedings of the International Conference Applications of Mathematics 2013, edited by J. Brandts, S. Korotov, M. Kř́ǐzek, J. Šístek, and T. Vejchodský (Institute of Mathematics, Academy of Sciences of the Czech Republic, 2013), pp. 104-116.

[9] J. Haslinger, V. Janovský, and T. Ligurský, Qualitative analysis of solutions to discrete static contact problems with Coulomb friction, Comput. Methods Appl. Mech. Engrg. 205-208, 149-161 (2012).

[10] J. Hughes and M. Friedman, A bisection-like algorithm for branch switching at a simple branch point, J. Sci. Comput. 41(1), 62-69 (2009).

[11] H. B. Keller, Lectures on Numerical Methods in Bifurcation Problems (Tata Institute of Fundamental Research; by SpringerVerlag, 1987), Notes by A. K. Nandakumaran and Mythily Ramaswamy.

[12] H. B. Khenous, J. Pommier, and Y. Renard, Hybrid discretization of the Signorini problem with Coulomb friction. Theoretical aspects and comparison of some numerical solvers, Appl. Numer. Math. 56(2), 163-192 (2006).

[13] A. Klarbring, Stability and critical points in large displacement frictionless contact problems, in: Friction and Instabilities, edited by J. A. C. Martins and M. Raous, CISM Courses and Lectures Vol. 457 (Springer-Verlag, Wien, 2002), pp. 39-64.

[14] T. Ligurský and Y. Renard, A continuation problem for computing solutions of discretised evolution problems with application to plane quasi-static contact problems with friction, Comput. Methods Appl. Mech. Engrg. 280, 222-262 (2014).

[15] T. Ligurský and Y. Renard, Bifurcations in piecewise-smooth steady-state problems: abstract study and application to plane contact problems with friction, Comput. Mech. 56(1), 39-62 (2015).

[16] A. Pinto da Costa and J. A. C. Martins, The evolution and rate problems and the computation of all possible evolutions in quasistatic frictional contact, Comput. Methods Appl. Mech. Engrg. 192(26-27), 2791-2821 (2003).

[17] Y. Renard and J. Pommier, Getfem++, An open source generic C++ library for finite element methods, http://home.gna . org/getfem.

[18] S. Scholtes, Introduction to piecewise differentiable equations, Preprint No. 53/1994, Institut für Statistik und Mathematische Wirtschaftstheorie, Universität Karlsruhe, 1994.

[19] M. Schulz and S. Pellegrino, Equilibrium paths of mechanical systems with unilateral constraints I. Theory, Proc. R. Soc. Lond. A 456(2001), 2223-2242 (2000). 